\title{
BMJ Open Impact of patient-centred and family- centred care meetings on intensive care and resource utilisation in patients with terminal illness: a single-centre retrospective observational study in Taiwan
}

Nin-Chieh Hsu, ${ }^{1}$ Chun-Che Huang, ${ }^{2}$ Wei-Chun Chen, ${ }^{3}$ Chong-Jen $\mathrm{Yu}^{4}$

To cite: Hsu N-C, Huang C-C, Chen W-C, et al. Impact of patient-centred and familycentred care meetings on intensive care and resource utilisation in patients with terminal illness: a single-centre retrospective observational study in Taiwan. BMJ Open 2019;9:e021561. doi:10.1136/ bmjopen-2018-021561

- Prepublication history for this paper is available online To view these files, please visit the journal online (http://dx.doi org/10.1136/bmjopen-2018021561).

$\mathrm{N}-\mathrm{CH}$ and $\mathrm{C}-\mathrm{CH}$ contributed equally.

Received 6 January 2018 Revised 14 November 2018 Accepted 20 December 2018

Check for updates

(C) Author(s) (or their employer(s)) 2019. Re-use permitted under CC BY-NC. No commercial re-use. See rights and permissions. Published by BMJ.

For numbered affiliations see end of article.

Correspondence to

Dr Nin-Chieh Hsu;

chesthsu@gmail.com

\section{ABSTRACT}

Objective Shared decision making is essential for patients and their families when facing serious and life-threatening diseases. This study aimed to evaluate the impact of patient-centred and family-centred care meetings (PFCCM) on intensive measures and resource utilisation during end-of-life (EOL) hospitalisation among terminally ill patients.

Design and setting A retrospective cross-sectional study using electronic medical records was conducted in a tertiary referral medical centre in Taiwan.

Participants We identified 6843 deceased patients with terminal illness who either received or did not receive PFCCM during EOL hospitalisation between January 2013 and December 2015.

Primary and secondary outcome measures Patients who were transferred to the intensive care unit (ICU). Those who underwent invasive or non-invasive mechanical ventilation, tracheostomy, haemodialysis and surgical intervention during the final hospitalisation were determined by the use of intensive care measures; secondary measures were individual total and daily medical expenditures. A generalised estimating equation (GEE) model was used to compare the differences between the two groups. OR and beta coefficients $(\beta)$ with $95 \% \mathrm{Cl}$ were estimated.

Results This study identified 459 patients (6.7\%) who received PFCCM during EOL hospitalisation. Multivariate analyses showed that patients who received PFCCM were less likely to have ICU admissions (OR $0.44,95 \% \mathrm{Cl} 0.34$ to 0.57$)$, undergo surgical interventions (OR $0.74,95 \% \mathrm{Cl}$ 0.58 to 0.95 ) and invasive mechanical ventilation (OR 0.50 , $95 \% \mathrm{Cl} 0.38$ to 0.66 ) during the final hospitalisation, after adjusting for patient demographics, clinical conditions and year of admission. Additionally, a significant decrease in daily medical expenditures was observed in PFCCM patients $(\beta-0.18,95 \% \mathrm{Cl}-0.25$ to -0.12$)$ than in nonPFCCM patients.

Conclusions Patient-physician discussion through PFCCM is associated with less intensive care utilisation and daily medical expenditure during EOL hospitalisation in terminally ill patients.
Strengths and limitations of this study

- This retrospective observational cross-sectional study sought to evaluate the impact of patient- and family-centred care meetings (PFCCM) on intensive care measures and resource utilisation during endof-life hospitalisation among terminally ill patients in Taiwan.

- Regression analyses with generalised estimating equation models were performed to analyse associations between PFCCM application and intensive care measures and resource utilisation.

- The generalisability of our results may be limited by the single-centre design restricted to deceased patients who died of a terminal illness.

\section{INTRODUCTION}

Excessive medical interventions that necessitate more effort and finances, with limited prospect of altering the ultimate clinical outcome, are defined as medical futility. ${ }^{1}$ In Taiwan's current healthcare system, terminally ill patients who are hospitalised may have a higher probability of undergoing excessive medical intervention during end-oflife (EOL) hospitalisation compared with patients in stable condition. Medical treatments and technology have advanced rapidly; in the process, certain terminally ill patients may undergo more aggressive and unnecessary medical intervention during the end stages of life. ${ }^{2}$ A previous study in Canada found that $35 \%$ of EOL patients received overtreatment, including unwanted cardiopulmonary resuscitation and other intensive care measures. ${ }^{3}$ An unrealistic family expectation for EOL patients to undergo an aggressive treatment was a significant predictor 
of prolonged and excessive intensive unit care without survival benefit. ${ }^{4}$

From the perspective of patients and their families, effective communication, shared decision making and relationships with clinicians and clinical teams are essential to providing quality EOL care. ${ }^{3}$ However, a recent systematic review revealed a lack of evidence supporting any kind of family meeting approach in an inpatient setting, ${ }^{5}$ although several prestudies and poststudies showed significant improvements in meeting family needs. ${ }^{6-8}$

The palliative care consultation service has been implemented in terminally ill patients for decades in hospitals in the USA. In Taiwan, the Ministry of Health and Welfare (MOHW) introduced palliative care regulations in $2000^{9}$; the palliative care consultation was designed to provide comprehensive EOL care for terminally ill patients, with qualified interdisciplinary specialists in acute care ward settings. ${ }^{10}$ In addition, the National Health Insurance Administration (NHIA) of the MOHW provided a fixed reimbursement of hospice care for both inpatient and home care. ${ }^{11}$ However, limited hospice resource and the patients' lack of awareness of their disease progression were major reasons for the slight increase in hospice utilisation from $11 \%$ in 2001 to $17 \%$ in $2006 .{ }^{10}$ After that, the NHIA incorporated palliative care family consultation into the medical insurance programme for terminally ill patients in December 2012. ${ }^{9}$ The attending physicians and patients or their families convened formal patientand family-centred care meetings (PFCCM) to explain disease status and prognosis, and to discuss the treatment options of EOL care and the preferred place of death.

To our knowledge, previous studies have suggested that the PFCCM could be associated with reduced aggressive life-sustaining treatments in terminally ill patients. ${ }^{3} 11$ Therefore, this study aimed to examine the influence of applying PFCCM on the use of intensive care measures and medical expenditures during EOL hospitalisation among terminally ill patients in Taiwan.

\section{METHODS}

\section{Study design and setting}

We conducted a retrospective cross-sectional study using data from electronic medical records between January 2013 and December 2015 from the National Taiwan University Hospital (NTUH), a 2000-bed tertiary referral medical centre in northern Taiwan. Confidentiality assurances were addressed by following the data regulations of the NTUH. We followed the Strengthening the Reporting of Observational Studies in Epidemiology guidelines for observational studies.

\section{Study subjects}

A total of 8199 deceased patients with terminal illness who were admitted to NTUH were identified between 2013 and 2015. We excluded patients whose length of hospital stay (LOS) was less than 48 hours $(n=637)$ and

$$
\begin{aligned}
& \text { Terminally ill patients who either died in } \\
& \text { hospital or were discharged to home to } \\
& \text { die during 2013-2015 ( } n=8199)
\end{aligned}
$$

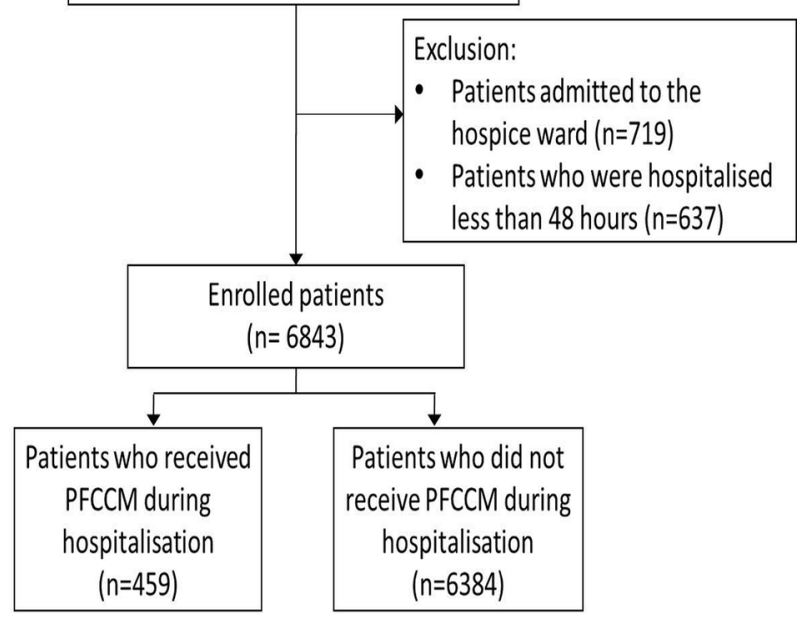

Figure 1 Flow diagram of study selection. PFCCM, patientcentred and family-centred care meetings.

who were admitted or transferred to the hospice ward $(n=719)$, because they did not receive PFCCM during their short stay. Patients who were reimbursed for hospice care before being referred to PFCCM were also excluded owing to the possible underestimation of their medical expenditure. ${ }^{11}$ The final sample comprised 6843 patients who did and did not receive PFCCM during hospitalisation. A flow diagram of study selection is shown in figure 1 .

\section{Variables}

The primary outcomes were the use of intensive care measures, including transferring to the intensive care unit (ICU) and undergoing invasive or non-invasive mechanical ventilation, tracheostomy, haemodialysis and surgical intervention during the final hospitalisation. We determined whether these intensive procedures were performed by selecting them as proxies of intensive care measures. The secondary outcome was the total and daily medical expenditures during the final hospitalisation, which represented hospital resource utilisation. Information on total medical expenditure was also gathered from electronic medical records. The total medical expenditure during the final hospitalisation for each deceased patient was measured from initial admission to death or discharge to die at home. Subsequently, to estimate average daily medical expenditure per patient, the total medical expenditure was divided by LOS.

The main independent variable was the status of patients who received PFCCM, which was defined as whether or not the terminally ill patients and their families had received PFCCM during EOL hospitalisation. Patients and/or their families who refused care by PFCCM were defined as those without receiving PFCCM. The PFCCM service was designed to provide comprehensive EOL care for terminally ill patients with qualified interdisciplinary specialists in acute care ward setting. ${ }^{9}$ 
PFCCM was conducted by attending physicians through formal discussions and shared decision-making practices with terminally ill patients and their families, regarding EOL-care goal, hospice care and preferred place of death. Terminally ill patients and families can choose to receive the PFCCM service and make decisions on EOL-care related preferences.

\section{Covariates}

The following covariates were included patient age and gender, catastrophic diseases covered by health insurance, main diagnoses, comorbidities, year of admission, LOS and disposition status. Under Taiwan's National Health Insurance scheme, patients who have cancers, chronic psychiatric diseases, haemodialysis or congenital disorders are eligible for catastrophic illness certificates after a review by the NHIA. Eligible patients with a catastrophic illness certificate are entitled to a waiver for copayments of related medical costs. Patients with a catastrophic illness have extremely high medical expenses in their final year of life, which has a potential impact on cost estimation. In addition, the diagnostic codes for each patient were classified according to the International Classification of Diseases, Clinical Modification, Ninth Revision (ICD-9-CM) coding system. We classified the main diagnoses into eight categories: infectious and parasitic diseases (ICD-9-CM codes 001-139); neoplasms (140-239); diseases of the circulatory system (390-459); diseases of the respiratory system (460-519); diseases of the digestive system (520-579); diseases of the genitourinary system (580-629); injury and poisoning (800-999) and other diagnoses. We applied the modified Charlson Comorbidity Index (CCI) to adjust the case mix for the severity of comorbid conditions during EOL hospitalisation. In addition, LOS was calculated as the total number of days of hospital stay, from admission to death or discharge. The disposition status of patients with terminal illness was classified broadly into death in hospital or discharge to die at home. Given the Chinese cultural meaning of dying at home, the numbers of deceased patients in Taiwan represent those patients commonly discharged against medical advice and often with artificial respiratory support to allow family members to keep the patient alive to die at home. ${ }^{12}$ Therefore, if the date of discharge for the final hospitalisation was also the date of death, the patient was recognised as dying in the hospital.

\section{Patient and public involvement statement}

Patients and public were not involved in this manuscript.

\section{Data analysis}

Demographics and outcomes, including intensive care procedures and medical expenditures between terminally ill patients who did and did not have PFCCM during the final hospitalisation were examined using the $\mathrm{x}^{2}$ or Fisher's exact tests for categorical variables and the MannWhitney U test for continuous variables.
In addition, univariate and multivariate regression analyses were performed to examine the associations between the receiving PFCCM status and outcomes. A GEE with an exchangeable correlation structure was applied to account for the clustering of patients hospitalised on the same index date. OR and beta coefficients with $95 \%$ CI of the status of receiving PFCCM, for the outcome variables, were estimated. All statistical analyses were conducted using SAS V.9.3 (SAS Institute), setting $\mathrm{p}<0.05$ as statistically significant.

\section{RESULTS}

During the study period, 6843 terminally ill patients were identified. Of these, 459 patients $(6.7 \%)$ received PFCCM during EOL hospitalisation. PFCCM intervention increased significantly from 2013 to 2015 ( $p<0.001$; table 1).

Table 1 depicts the patient demographics, clinical conditions and outcomes in terms of intensive care measures and resource utilisation between patients who did and did not receive PFCCM. The distribution of age was similar between the two groups, with more than half $(54.2 \%)$ of the patients aged above 65 years. Male patients $(52.3 \%)$ received PFCCM slightly less than their female counterparts $(59.3 \%)$ did $(\mathrm{p}=0.009)$. However, we observed that among patients who had catastrophic diseases, a neoplasm diagnosis and CCI $\geq 5$ (ie, more severe comorbid conditions), there were more patients who received PFCCM that those who did not. In addition, LOS was longer in patients who received PFCCM than in those who did not (31.1 days vs 27.4 days, $\mathrm{p}<0.001)$. The disposition pattern between the two groups was similar (table 1).

In terms of intensive care measures and resource utilisation, the crude rates of transferring to the ICU and undergoing surgery and invasive mechanical ventilation, as well as the daily medical expenditures during the final hospitalisation, were lower among patients who received PFCCM than in those who did not (table 2). We observed less frequent non-invasive ventilation, tracheostomy and haemodialysis, as well as lower total medical expenditures, in patients who received PFCCM, although the differences were not significant statistically. However, a boxplot showed that patients who received PFCCM had lower median daily medical expenditures compared with those who did not (Taiwan dollars (TWD) 7878.2 vs TWD 9610.9, $\mathrm{p}<0.001$; figure 2). In addition, after excluding extreme outliers for 14 non-PFCCM patients with daily medical expenditure more than TWD 200000 (see the right most boxplot of figure 2), the results remain similar to those obtained before (data not shown).

After we controlled for patient demographics and clinical conditions, multivariate GEE analyses showed that patients who received PFCCM, compared with those who did not, were less likely to have ICU admissions (OR 0.44, $95 \%$ CI 0.34 to 0.57 ), and to undergo surgery (OR 0.74 , $95 \%$ CI 0.58 to 0.95 ) and invasive mechanical ventilation 
Table 1 Characteristics of patients with and without receiving PFCCM

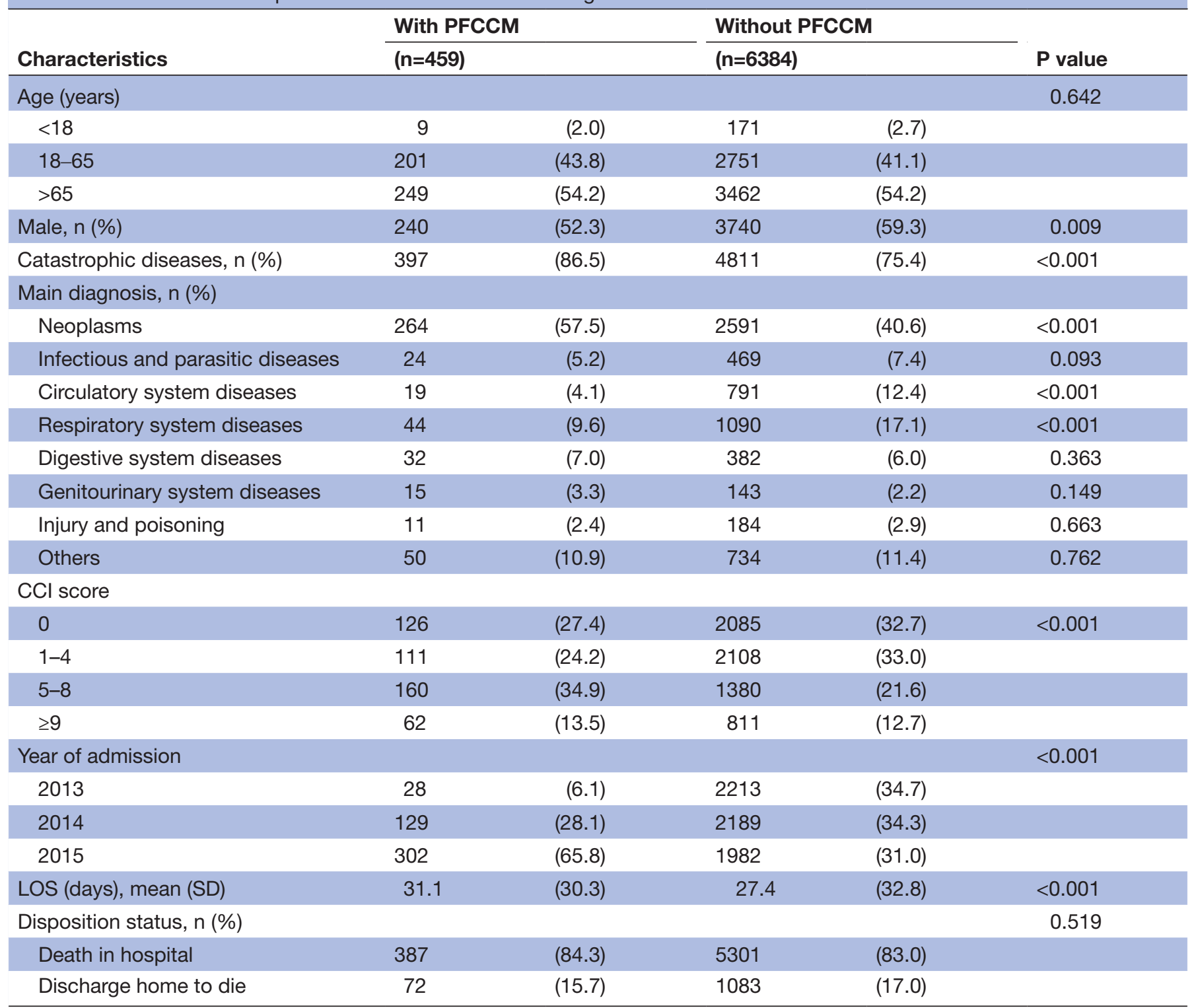

Values are expressed as number (\%) or mean \pm SD.

$\mathrm{CCl}$, Charlson Comorbidity Index; LOS, length of hospital stay; PFCCM, patient-centred and family-centred care meetings.

(OR 0.50 , 95\% CI 0.38 to 0.66 ) during the final hospitalisation. The use of non-invasive ventilation, tracheostomy and haemodialysis showed no significant difference. Moreover, after covariate adjustment, we observed associated reductions in daily medical expenditures $(\beta-0.18$, $95 \%$ CI -0.25 to -0.12 ) in patients who received PFCCM (table 3).

\section{DISCUSSION}

This is the first study to evaluate the influence of PFCCM on intensive care and resource utilisation during the final hospitalisation of terminally ill patients in non-hospice settings. We found that patients who received PFCCM were less likely to have ICU admissions (56\%), to undergo surgical procedures (26\%) and invasive mechanical ventilation (50\%) during the final hospitalisation, even after adjusting for relevant covariates. Additionally, for patients who received PFCCM, their daily medical expenditure during the final hospitalisation decreased.

Certain aggressive care measures at the EOL, including intensive, high-tech and often unproven therapies may be associated negatively with patients' preferences for life-sustaining treatments. For example, a previous study found that the best predictor of a prolonged ICU stay in patients who were unlikely to survive was 'unrealistic family expectations, ${ }^{4}$; these expectations are likely to result in aggressive intensive care for patients and unfavourable experiences for their family, both of which are harmful. Less aggressive intensive care at the EOL is beneficial for patients and has been associated with a 
Table 2 Use of intensive care measures and medical expenditures during the final hospitalisation between patients with and without receiving PFCCM

\begin{tabular}{|c|c|c|c|c|c|}
\hline & \multicolumn{2}{|c|}{ With PFCCM } & \multicolumn{2}{|c|}{ Without PFCCM } & \multirow[b]{2}{*}{ P value } \\
\hline & $(n=459)$ & & $(n=6384)$ & & \\
\hline ICU admission, n (\%) & 100 & $(21.8)$ & 2773 & $(43.4)$ & $<0.001$ \\
\hline Invasive mechanical ventilation, n (\%) & 90 & $(19.6)$ & 2402 & $(37.6)$ & $<0.001$ \\
\hline Non-invasive ventilation, $\mathrm{n}(\%)$ & 52 & $(11.3)$ & 841 & $(13.2)$ & 0.282 \\
\hline Haemodialysis, n (\%) & 47 & $(10.2)$ & 846 & $(13.3)$ & 0.073 \\
\hline Total medical expenditure (TWD), mean (SD) & 344423.6 & (538 058) & 387106.2 & $(635660)$ & 0.438 \\
\hline Daily medical expenditure* (TWD), mean (SD) & 10104.6 & $(7157)$ & 16013.6 & $(23727)$ & $<0.001$ \\
\hline
\end{tabular}

${ }^{*}$ The daily medical expenditures per patient were calculated as the total medical expenditures divided by the length of the hospital stay. ICU, intensive care unit; PFCCM, patient-centred and family-centred care meetings; TWD, Taiwan dollars.

lower risk for psychiatric morbidity and lesser regrets for bereaved family caregivers. ${ }^{13}$

The study to understand prognoses and preferences for outcomes and risks of treatments trial in the USA reported shortcomings in EOL communications and prolonged ICU admissions at the EOL. ${ }^{14}$ However, interventions that focused on physicians, nurses and other clinicians have failed to improve patient care or reduce negative outcomes in the same trial. Patients have experienced no improvement in patient-physician communication through these medical interventions, which also have not reduced the use of hospital resources. ${ }^{14}$ However, the reasons of ineffective communication remained unanswered. This finding is consistent with previous studies ${ }^{15-17}$ supporting that adequate provision

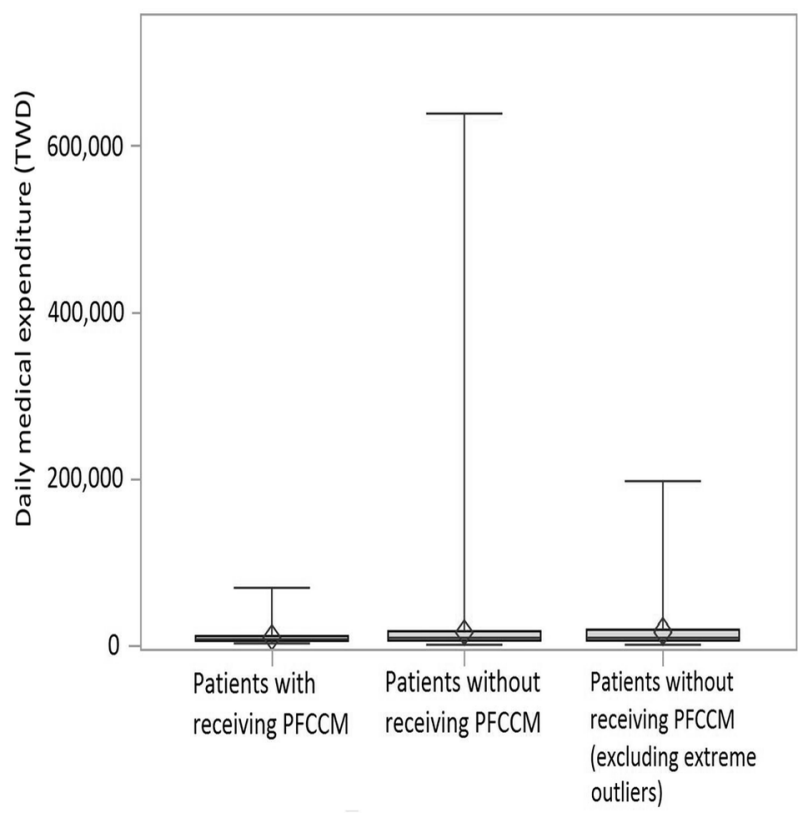

Figure 2 Daily medical expenditure during the final hospitalisation among patients with and without receiving patient- and family-centred care meetings (PFCCM) (excluding extreme outliers for non-PFCCM patients). of PFCCM may curb medical expenditures. Most studies have focused on interventions by palliative consultation teams with patients, ${ }^{13}{ }^{18-20}$ but the results regarding interventions with families have been inconclusive. ${ }^{5}$ The family perspective on EOL care revealed the need for communication with physicians ${ }^{21-23}$; moreover, encouraging family participation in EOL discussions, and understanding their burdens and supporting their needs must be the main tasks and purposes of PFCCM. ${ }^{24-26}$

Although several studies have reported that palliative care consultation can decrease hospital costs, ${ }^{15} 162728$ evidence of medical futility at EOL has remained. In a recent report from three US hospitals, the researchers found that lower hospital costs following palliative care consultations resulted mainly from shorter LOS rather than fewer high-intensity treatments. ${ }^{29}$ This finding implies that goals of care and treatment preference discussions are necessary to minimise inappropriate treatments at the EOL. The economic impact of interventions on families and caregivers was an important but underexamined issue. ${ }^{30}$ In this study, involving family caregivers in medical decision making through PFCCM for terminally ill patients may potentially reduce ICU admissions, surgery and invasive mechanical ventilation, and lower daily medical expenditures during the final hospitalisation. This study suggests that shared decision making between patients, families and clinicians may minimise unnecessary medical and surgical interventions at the EOL. Previous research has reported that higher medical expenditures are not associated with better outcomes for the elderly, ${ }^{31}$ and avoiding futile medical and surgical treatments for terminally ill patients may become even more important when unwanted adverse effects might occur.

Emotional support, shared decision-making and respect are essential in providing quality EOL care. ${ }^{23}$ However, fear of affecting patients' emotional coping and hope is a barrier for physicians to engage in EOL discussions. ${ }^{32}$ In addition, patients' and families' views of EOL care differ, 
Table 3 Univariate and multivariate analyses for intensive care measures and medical expenditures during the final hospitalisation among patients with receiving patient- and family-centred care meetings

\begin{tabular}{|c|c|c|c|c|c|c|}
\hline & \multicolumn{3}{|c|}{ Univariate analysis } & \multicolumn{3}{|c|}{ Multivariate analysis } \\
\hline & OR & $(95 \% \mathrm{Cl})$ & $P$ value & OR & $(95 \% \mathrm{Cl})$ & $P$ value \\
\hline ICU admission & 0.36 & (0.29 to 0.45$)$ & $<0.001$ & $0.44^{*}$ & (0.34 to 0.57$)$ & $<0.001$ \\
\hline Invasive mechanical ventilation & 0.40 & (0.32 to 0.51$)$ & $<0.001$ & $0.50^{*}$ & (0.38 to 0.66$)$ & $<0.001$ \\
\hline Non-invasive ventilation & 0.85 & $(0.63$ to 1.14$)$ & 0.269 & $0.96^{*}$ & (0.70 to 1.31$)$ & 0.785 \\
\hline \multirow[t]{2}{*}{ Haemodialysis } & 0.75 & (0.55 to 1.01$)$ & 0.062 & $0.84^{*}$ & (0.59 to 1.17$)$ & 0.302 \\
\hline & $\boldsymbol{\beta}$ & $(95 \% \mathrm{Cl})$ & $P$ value & $\boldsymbol{\beta}$ & $(95 \% \mathrm{Cl})$ & $P$ value \\
\hline Total medical expenditure (TWD) & -0.16 & $(-0.26$ to 0.03$)$ & 0.117 & $-0.01 \dagger$ & $(-0.10$ to 0.16$)$ & 0.600 \\
\hline Daily medical expenditure (TWD) & -0.46 & $(-0.53$ to -0.39$)$ & $<0.001$ & $-0.18 \dagger$ & $(-0.25$ to -0.12$)$ & $<0.001$ \\
\hline
\end{tabular}

*Adjusted for patient's age, gender, catastrophic diseases, main diagnoses, Charlson Comorbidity Index score, LOS, disposition status and year of admission as compared with patients without receiving PFCCM.

†Adjusted for patient's age, gender, catastrophic diseases, main diagnoses, Charlson Comorbidity Index score, disposition status and year of admission as compared with patients without receiving PFCCM.

ICU, intensive care unit; PFCCM, patient-centred and family-centred care meetings; TWD, Taiwan dollars.

and EOL discussions that involve both patients and families may, therefore, be necessary to resolve conflicts. ${ }^{21}$ There is a general agreement that the patient and family represent the unit of care in palliative care settings, and the implementation of PFCCM aimed to deliver appropriate services to patients and to understand their disease status, emotional experiences, financial burdens and unmet needs. ${ }^{2633}$ A previous study showed that the rating of patient-centred goals increase fivefold when family caregivers are involved in interdisciplinary care interventions for terminally ill patients. ${ }^{33}$ Thus, we believe that PFCCM provision during EOL hospitalisation may be essential for terminally ill patients.

This study provides a comprehensive comparison of the intensive care measures and resource utilisation during the final hospitalisation in terminally ill patients with and without receiving PFCCM. The PFCCM meetings are formal meetings that are reimbursable by the Taiwan's National Health Insurance when records and documents are produced. Such meetings were proposed, prepared and conducted by the attending physician in charge whether in the ward or ICU setting. Despite no difference in non-invasive ventilation, tracheostomy and haemodialysis, a lower probability of having ICU admission, undergoing surgical interventions and invasive mechanical ventilation was observed among patients with receiving PFCCM. In addition, while previous literature focuses on the impact of palliative care family consultation on terminally ill cancer patients, this study included patients dying from both cancer and non-cancer diagnoses and was conducted in acute care ward settings.

This study has several limitations. First, the small number of terminally ill patients who actually received PFCCM might have introduced a selection bias. The small number may be due to the patients' receptiveness to palliative care or advance care planning. Second, terminally ill patients who received PFCCM and survived during the final hospitalisation were not included. Third, family meetings that are organised and facilitated by a trained palliative medicine consultant team using a consistent structure and approach will predict greater success than informal meetings at the patient's bedside led by a healthcare professional such as a physician or nurse practitioner in a specialist consultant team. In addition, the widely divergent approaches of family meetings may have different outcomes. Fourth, the sample included only terminally ill patients from one medical centre, which may limit the generalisability of the results to other medical centres and community hospitals.

\section{CONCLUSIONS}

Shared decision making through PFCCM meetings during EOL hospitalisation is associated with lower likelihood of transferring to the ICU and of undergoing surgery and invasive mechanical ventilation in terminally ill patients. We also observed an associated reduction in the daily medical expenditures in patients who received PFCCM. This finding suggests that the provision of PFCCM could potentially reduce inappropriate intensive care measures and resource utilisation before terminally ill patients die. In addition, PFCCM allow patients and families to play an active role in the shared decision making on aggressive intensive care treatments. Further research could help identify the key components of PFCCM in EOL care that may be critical to success in assisting patients and families facing the imminent death of a loved one. 
Author affiliations

${ }^{1}$ Division of Hospital Medicine, Department of Internal Medicine, National Taiwan Uinversity Hospital, Taipei, Taiwan

${ }^{2}$ Department of Medical Research, Taichung Veterans General Hospital, Taichung, Taiwan

${ }^{3}$ Medical Affairs Office, National Taiwan University Hospital, Taipei, Taiwan ${ }^{4}$ Department of Internal Medicine, National Taiwan University Hospital, Taipei, Taiwan

Acknowledgements We appreciate the assistance from the Department of Medical Research of the National Taiwan University Hospital.

Contributors $\mathrm{N}-\mathrm{CH}$ designed the study and drafted the manuscript. C-CH analysed and interpreted the results, and provided critical review of the manuscript. W-CC led the data collection. C-JY gave critical revision of the manuscript. All authors drafted the manuscript and were part of the revision process. All authors agreed to the final version of the manuscript before submission.

Funding The authors have not declared a specific grant for this research from any funding agency in the public, commercial or not-for-profit sectors.

Competing interests None declared.

Patient consent for publication Not required

Ethics approval This study was approved by the Research Ethics Committee of the National Taiwan University Hospital (201502011RINC), which waived the need for written informed consent from the participants, since the data were analysed anonymously.

Provenance and peer review Not commissioned; externally peer reviewed.

Data sharing statement № additional data available.

Open access This is an open access article distributed in accordance with the Creative Commons Attribution Non Commercial (CC BY-NC 4.0) license, which permits others to distribute, remix, adapt, build upon this work non-commercially, and license their derivative works on different terms, provided the original work is properly cited, appropriate credit is given, any changes made indicated, and the use is non-commercial. See: http://creativecommons.org/licenses/by-nc/4.0/.

\section{REFERENCES}

1. Swetz KM, Burkle CM, Berge $\mathrm{KH}$, et al. Ten common questions (and their answers) on medical futility. Mayo Clin Proc 2014;89:943-59.

2. Mueller PS, Hook CC. Technological and treatment imperatives, life-sustaining technologies, and associated ethical and social challenges. Mayo Clin Proc 2013;88:641-4.

3. Heyland DK, Ilan R, Jiang X, et al. The prevalence of medical error related to end-of-life communication in Canadian hospitals: results of a multicentre observational study. BMJ Qual Saf 2016;25:671-9.

4. Berge KH, Maiers DR, Schreiner DP, et al. Resource utilization and outcome in gravely ill intensive care unit patients with predicted inhospital mortality rates of $95 \%$ or higher by APACHE III scores: the relationship with physician and family expectations. Mayo Clin Proc 2005;80:166-73.

5. Cahill PJ, Lobb EA, Sanderson C, et al. What is the evidence for conducting palliative care family meetings? A systematic review. Palliat Med 2017;31:197-211.

6. Fukui M, Iwase S, Sakata N, et al. Effectiveness of using clinical guidelines for conducting palliative care family meetings in Japan. Support Care Cancer 2013;21:53-8.

7. Hannon B, O'Reilly V, Bennett K, et al. Meeting the family: measuring effectiveness of family meetings in a specialist inpatient palliative care unit. Palliat Support Care 2012;10:43-9.

8. Hudson P, Thomas T, Quinn K, et al. Family meetings in palliative care: are they effective? Palliat Med 2009;23:150-7.

9. Ministry of Health and Welfare. The Ministry of Health and Welfare ( $\mathrm{MOHW}$ ) calls on respecting the lives and medical care discretion of the terminally ill. http://www.mohw.gov.tw/cp-199-482-2.html (accessed 15 Sep 2018).

10. Lu CY, Shen WC, Kao CY, et al. Impact of palliative care consultation service on terminally ill cancer patients: a 9-year observational cohort study in Taiwan. Medicine 2016;95:e2981.
11. Chiang JK, Kao YH, Lai NS. The impact of hospice care on survival and healthcare costs for patients with lung cancer: a national longitudinal population-based study in Taiwan. PLoS One 2015;10:e0138773

12. Tang ST, Sc W, Hung YN, et al. Trends in quality of end-of-life care for Taiwanese cancer patients who died in 2000-2006. Ann Oncol 2009;10:343-8.

13. Wright $A A$, Zhang $B$, Ray $A$, et al. Associations between end-of-life discussions, patient mental health, medical care near death, and caregiver bereavement adjustment. JAMA 2008;300:1665-73.

14. Connors Jr AF, Dawson NV, Desbiens NA, et al. A controlled trial to improve care for seriously ill hospitalized patients. the study to understand prognoses and preferences for outcomes and risks of treatments (support). the support principal investigators. JAMA 1995;274:1591-8.

15. Morrison RS, Dietrich J, Ladwig S, et al. Palliative care consultation teams cut hospital costs for Medicaid beneficiaries. Health Aff 2011;30:454-63.

16. Morrison RS, Penrod JD, Cassel JB, et al. Cost savings associated with US hospital palliative care consultation programs. Arch Intern Med 2008;168:1783-90.

17. O'Mahony S, McHenry J, Blank AE, et al. Preliminary report of the integration of a palliative care team into an intensive care unit. Palliat Med 2010;24:154-65.

18. Oczkowski SJ, Chung HO, Hanvey L, et al. Communication tools for end-of-life decision-making in the intensive care unit: a systematic review and meta-analysis. Crit Care 2016;20:97.

19. Lo B, Quill T, Tulsky J. Discussing palliative care with patients. acp-asim end-of-life care consensus panel. american college of physicians-american society of internal medicine. Ann Intern Med 1999;130:744-9.

20. Tulsky JA, Arnold RM, Alexander SC, et al. Enhancing communication between oncologists and patients with a computerbased training program: a randomized trial. Ann Intern Med 2011;155:593-601.

21. Steinhauser KE, Christakis NA, Clipp EC, et al. Factors considered important at the end of life by patients, family, physicians, and other care providers. JAMA 2000;284:2476-82.

22. Tang ST, Li CY, Liao YC. Factors associated with depressive distress among Taiwanese family caregivers of cancer patients at the end of life. Palliat Med 2007;21:249-57.

23. Teno JM, Clarridge BR, Casey V, et al. Family perspectives on endof-life care at the last place of care. JAMA 2004;291:88-93.

24. Aoun S, Deas K, Toye C, et al. Supporting family caregivers to identify their own needs in end-of-life care: Qualitative findings from a stepped wedge cluster trial. Palliat Med 2015;29:508-17.

25. Emanuel EJ, Fairclough DL, Slutsman J, et al. Understanding economic and other burdens of terminal illness: the experience of patients and their caregivers. Ann Intern Med 2000;132:451-9.

26. Harding R, Higginson IJ. What is the best way to help caregivers in cancer and palliative care? A systematic literature review of interventions and their effectiveness. Palliat Med 2003;17:63-74.

27. Gade G, Venohr I, Conner D, et al. Impact of an inpatient palliative care team: a randomized control trial. J Palliat Med 2008;11:180-90.

28. Penrod JD, Deb P, Dellenbaugh C, et al. Hospital-based palliative care consultation: effects on hospital cost. J Palliat Med 2010;13:973-9.

29. May P, Garrido MM, Cassel JB, et al. Cost analysis of a prospective multi-site cohort study of palliative care consultation teams for adults with advanced cancer: Where do cost-savings come from? Palliat Med 2017;31:378-86.

30. May P, Morrison RS, Murtagh FE. Current state of the economics of palliative and end-of-life care: A clinical view. Palliat Med 2017;31:293-5.

31. Tsugawa Y, Jha AK, Newhouse JP, et al. Variation in physician spending and association with patient outcomes. JAMA Intern Med 2017; 177:675-82.

32. Mack JW, Smith TJ. Reasons why physicians do not have discussions about poor prognosis, why it matters, and what can be improved. J Clin Oncol 2012;30:2715-7.

33. Wittenberg-Lyles E, Oliver DP, Demiris G, et al. Inviting the absent members: examining how caregivers' participation affects hospice team communication. Palliat Med 2010;24:192-5. 